\title{
SOME OPEN PROBLEMS RELATED TO CREATIVE TELESCOPING
}

\author{
SHAOSHI CHEN* AND MANUEL KAUERS*
}

\begin{abstract}
Creative telescoping is the method of choice for obtaining information about definite sums or integrals. It has been intensively studied since the early 1990s, and can now be considered as a classical technique in computer algebra. At the same time, it is still subject of ongoing research. In this paper, we present a selection of open problems in this context. We would be curious to hear about any substantial progress on any of these problems.
\end{abstract}

\section{INTRODUCTION}

Summation problems arise in all areas of mathematics, especially in discrete mathematics and combinatorics. The general task is to compute for a given expression describing a summand sequence $f(n, k)$ an expression that describes the sum sequence $F(n)=\sum_{k=0}^{n} f(n, k)$. Depending on the type of expressions allowed for summand and/or sum, a solution may or may not exist. The classical class of expressions considered in the theory of symbolic summation is the class of hypergeometric terms. A univariate sequence $f(k)$ is called hypergeometric if the shift quotient $f(k+1) / f(k)$ can be simplified to a rational function in $k$. For example, $f(k)=k$ ! is hypergeometric because $f(k+1) / f(k)=k+1$ is a polynomial. Another example is $f(k)=2^{k}$. Gosper's algorithm [49] solves the decision problem for hypergeometric summation: given a hypergeometric term $f(k)$ (i.e., given a rational function $r(k)$ such that $f(k+1) / f(k)=r(k)$ ), it computes a hypergeometric term $F(k)$ such that $F(k+1)-F(k)=f(k+1)$, or it certifies that no such hypergeometric term exists. When $F(k)$ is found, it implies the closed form representation $\sum_{k=0}^{n} f(k)=F(n+1)-F(0)$. For example, Gosper's algorithm can find the formula $\sum_{k=0}^{n} k k !=(n+1) !-1$.

Gosper's algorithm only applies to so-called indefinite sums. These are sums in which the upper summation bound is a variable that does not occur in the summand expression. All other sums are called definite. For example, $\sum_{k=0}^{n}\left(\begin{array}{c}m \\ k\end{array}\right)$ is an indefinite sum (involving a parameter $m$ ), while $\sum_{k=0}^{n}\left(\begin{array}{l}n \\ k\end{array}\right)$ is a definite sum. The distinction is important because there does exist a closed form for the latter sum (it is equal to the nice expression $2^{n}$ ), but no closed form exist when $m$ and $n$ are unrelated.

In order to process definite sums, we can use the technique of creative telescoping. Informally, creative telescoping solves the following problem: Given an expression $f(n, k)$, it computes polynomials $c_{0}(n), \ldots, c_{r}(n)$, not all zero, and an expression $g(n, k)$, such that

$$
c_{0}(n) f(n, k)+\cdots+c_{r}(n) f(n+r, k)=g(n, k+1)-g(n, k) .
$$

When such a relation is available, we can sum it for $k$ from 0 to $n$ to obtain a relation of the form

$$
c_{0}(n) F(n)+\cdots+c_{r}(n) F(n+r)=G(n)
$$

for the definite sum $F(n)=\sum_{k=0}^{n} f(n, k)$ and some explicit expression $G(n)$. From such an equation, other algorithms can be used to find closed form representations for $F(n)$ (or prove that there are none), or information about its asymptotic behaviour for $n \rightarrow \infty$, or to compute a large number of terms of the sequence efficiently.

* Supported by the NSFC grant 11501552 and by the President Fund of the Academy of Mathematics and Systems Science, CAS (2014-cjrwlzx-chshsh). This work was also supported by the Fields Institute's 2015 Thematic Program on Computer Algebra.

* Supported by the Austrian FWF grants F5004, Y464-N18, and W1214. 
The method of creative telescoping was propagated by Zeilberger in the early 1990s [97, 96, 100, 73] (although the word "creative telescoping" already appears in [91]). Zeilberger also gave the first algorithm for creative telescoping applicable to hypergeometric terms. This algorithm, now known as Zeilberger's algorithm, is a clever modification of Gosper's algorithm. Zeilberger also formulated a vision for doing creative telescoping in the much more general realm of holonomic functions [99]. Over the years, this led to the development of operator-based techniques such as Chyzak's algorithm [37, 38] as well as difference-field-based techniques mainly developed by Schneider [84, 86, 87].

Ore algebras provide a setting in which the creative telescoping problem can be formulated in great generality. To give an idea, let us consider the case where $C$ is a field of characteristic zero, $K=C(n, k)$ is the field of rational functions in $n$ and $k$ with coefficients in $C$, and $A=K\left[S_{n}, S_{k}\right]$ is the polynomial ring in two variables $S_{n}, S_{k}$ with coefficients in $K$. The multiplication on $A$ is defined in such a way that we have $S_{n} S_{k}=S_{k} S_{n}$ and $S_{n} r(n, k)=r(n+1, k) S_{n}$ and $S_{k} r(n, k)=$ $r(n, k+1) S_{k}$ for all $r \in K$. The elements of $A$ can then be viewed as operators that act on a space $F$ of bivariate sequences. For any particular sequence $f \in F$, we may then consider the left ideal $\operatorname{ann}(f)=\{L \in A: L \cdot f=0\}$ of all the operators in $A$ which map $f$ to zero. Then the problem of creative telescoping is to find some operator $P \in C(n)\left[S_{n}\right] \backslash\{0\}$ and some operator $Q \in A$ such that $P-\left(S_{k}-1\right) Q \in \operatorname{ann}(f)$. In such a representation, $P$ is called a telescoper for $f$ and $Q$ is called a certificate for $P$.

There are some other flavors of the creative telescoping problem which are also of interest. In particular, there is a differential version, which is useful for integration. In this case, we consider the Ore algebra $A=C(x, y)\left[D_{x}, D_{y}\right]$ consisting of all linear differential operators with coefficients in $C(x, y)$, acting on a space $F$ of bivariate functions. Note that the multiplication laws for differential operators are slightly different from the multiplication laws for recurrence operators: here we have $D_{x} D_{y}=D_{y} D_{x}$ and $D_{x} a=a D_{x}+\frac{d}{d x} a$ and $D_{y} a=a D_{y}+\frac{d}{d y} a$ for all $a \in C(x, y)$. For any particular function $f \in F$, let $\operatorname{ann}(f)=\{L \in A: L \cdot f=0\}$ again denote the left ideal consisting of all the operators in $A$ that map $f$ to zero. The problem of creative telescoping is then to find some operator $P \in C(x)\left[D_{x}\right] \backslash\{0\}$ and some operator $Q \in A$ such that $P-D_{y} Q \in \operatorname{ann}(f)$. In the context of integration, such an operator can serve the same purpose as a creative telescoping relation of the form discussed before in the context of summation: From $\left(P-D_{y} Q\right) \cdot f=0$ follows $0=\int_{0}^{1}\left(\left(P-D_{y} Q\right) \cdot f\right)(x, y) d y=P \cdot \int_{0}^{1} f(x, y) d y-[(Q \cdot f)(x, y)]_{y=0}^{1}$, so we have $P \cdot F(x)=G(x)$ for $F(x)=\int_{0}^{1} f(x, y) d y$ and some simple and explicit function $G(x)$.

A lot of research has been done on algorithms for creative telescoping during the past 25 years. A reasonably complete and almost up-to-date overview of the state of the art is given in Chyzak's Habilitation thesis from 2014 [39]. The focus of this thesis is on the algorithmic aspects and the theoretical foundations. In addition, there are many papers that implicitly or explicitly make use of the theory by simplifying sums or integrals using computer programs based on the method of creative telescoping. This underlines the importance of the method. At the same time, despite the successful work on creative telescoping that has been done in the past, there is still a number of open problems which do not yet have satisfactory answers. In the present article, we offer a collection of such open problems. The choice is obviously biased by our personal interests. However, we believe that significant progress on any of these problems would be a valuable contribution to the advance of symbolic summation.

\section{Reduction-Based Algorithms}

Algorithms for creative telescoping can be distinguished according to their input class or according to the algorithmic technique they are based on. The available algorithmic techniques can be divided into four generations of creative telescoping algorithms. Algorithms from the first generation use elimination theory for operator ideals [46, 88, 89, 73, 95, 41]. Zeilberger's algorithm from 1990 [98] and its generalizations $[9,38,61,87]$ form the second generation. The third generation is based on an idea that was first formulated by Apagodu and Zeilberger [71, 10] and has later been refined and generalized [64, 27, 26, 28]. Algorithms from the fourth and most recent generation of 
creative telescoping algorithms are called reduction-based algorithms. They were first introduced by Bostan et al. [14] for integration of rational functions. The basic idea is as follows. Consider a rational function $f=p / q \in C(x, y)$. The task is to find $c_{0}, \ldots, c_{r} \in C(x)$ such that there exists $g \in C(x, y)$ with

$$
c_{0} f+c_{1} D_{x} f+\cdots+c_{r} D_{x}^{r} f=D_{y} g .
$$

Consider the partial derivatives $f, D_{x} f, D_{x}^{2} f, \cdots \in C(x, y)$. Using Hermite reduction, we can write each of them in the form $D_{x}^{i} f=D_{y} g_{i}+h_{i}$ for some $g_{i}, h_{i} \in C(x, y)$ where $h_{i}$ has a square free denominator whose degree exceeds the degree of its numerator. The denominators of all these $h_{i}$ divide the square free part of the denominator of $f$ in $C(x)[y]$, so the $C(x)$-subspace of $C(x, y)$ generated by $h_{0}, h_{1}, \ldots$ has finite dimension. If the dimension is $r$, then we can find $c_{0}, \ldots, c_{r} \in C(x)$, not all zero, such that $c_{0} h_{0}+\cdots+c_{r} h_{r}=0$. For these $c_{0}, \ldots, c_{r}$ we then have

$$
c_{0} f+c_{1} D_{x} f+\cdots+c_{r} D_{x}^{r}=D_{y}\left(g_{0}+g_{1}+\cdots+g_{r}\right)+0,
$$

as desired.

The approach is not limited to rational functions and has been generalized to hyperexponential terms [15], hypergeometric terms (for the summation case) [25, 55] and algebraic functions [29]. It has also been worked out for the mixed case when the integrand is a hypergeometric-hyperexponential term $f_{n}(x)$ [17], and it is being worked out by Du, Huang and Li [43] the $q$-case. At this stage, the summation case for hypergeometric-hyperexponential terms $f_{n}(x)$ is still open, so this shall be our first problem.

Problem 1. Develop a reduction based creative telescoping algorithm which for a given hypergeometric-hyperexponential term $f_{n}(x)$ computes, if possible, rational functions $c_{0}, \ldots, c_{r} \in C(x)$, not all zero, such that there exists a hypergeometric-hyperexponential term $g_{n}(x)$ with

$$
c_{0} f_{n}(x)+\cdots+c_{r} D_{x}^{r} f_{n}(x)=g_{n+1}(x)-g_{n}(x) .
$$

In the pure differential case, we could consider integrands from larger classes of functions. The largest class considered so far was the class of algebraic functions [29]. It is based on Trager's Hermite reduction [90, 20]. The correctness of the method relies heavily on Chevalley's theorem [35], according to which any non-constant algebraic function must have a pole at some place (possibly over infinity). Since there is no analogous theorem for general D-finite functions, not even for solutions of Fuchsian equations, it is not clear how to generalize the reduction based algorithm from algebraic functions to (Fuchsian) D-finite functions. This is our second problem.

Problem 2. Develop a reduction based creative telescoping algorithm which for a given (Fuchsian) $D$-finite function $f(x, y)$ computes, if possible, rational functions $c_{0}, \ldots, c_{r} \in C(x)$, not all zero, such that there exists an operator $Q \in C(x, y)\left[D_{x}, D_{y}\right]$ with $\left(c_{0}+c_{1} D_{x}+\cdots+c_{r} D_{x}^{r}\right) \cdot f=D_{y} Q \cdot f$.

\section{Order-Degree Curves}

When a function admits a telescoper, the telescoper is not uniquely determined. The set of telescopers rather forms a left ideal in the operator algebra $C(x)\left[D_{x}\right]$ (or in $C(n)\left[S_{n}\right]$, respectively). Since the operator algebras $C(x)\left[D_{x}\right]$ and $C(n)\left[S_{n}\right]$ are left-Euclidean domains, it follows that there is a unique monic telescoper of minimal possible order - called the minimal telescoper - and that all the other telescopers are left-multiples of this telescoper.

For the purpose of estimating the computational cost of creative telescoping algorithms, it is interesting to know bounds for the size of telescopers relative to characteristic parameters of the input. Besides bounds on the order $r$ of the telescopers, it is also of interest to bound the sizes of its coefficients. After clearing denominators (from left), we can assume that the telescoper lives in $C[x]\left[D_{x}\right]$ or $C[n]\left[S_{n}\right]$, and we can ask for its degree $d$ with respect to $x$ or $n$.

Unlike $C(x)\left[D_{x}\right]$ and $C(n)\left[S_{n}\right]$, the rings $C[x]\left[D_{x}\right]$ and $C[n]\left[S_{n}\right]$ are not left-principal. As a consequence, we can in general not minimize the order $r$ and the degree $d$ simultaneously. Instead, we must expect that telescopers of low order $r$ have a high degree $d$ and telescopers of low degree $d$ have high order $r$. To describe the general situation, we use a function $c: \mathbb{R} \rightarrow \mathbb{R}$ such that for 
each $r \geq r_{\min } \in \mathbb{N}$ there is a telescoper of order $r$ and degree at most $c(r)$. The graph of the function $c$ is called an order-degree curve for the summation/integration problem at hand.

It turns out that order-degree curves can be derived from the Apagodu-Zeilberger algorithm [71]. Apagodu and Zeilberger used their approach to derive bounds on the order of the telescopers. Again, the idea is easily explained for the case of rational functions. Consider $f=p / q \in C(x, y)$ and suppose for simplicity that $\operatorname{deg}_{y} p<\operatorname{deg}_{y} q$. By induction, it can be shown that $D_{x}^{i} f=p_{i} / q^{i+1}$ for some polynomial $p_{i} \in C(x)[y]$ of degree at most $\operatorname{deg}_{y} p_{i} \leq \operatorname{deg}_{y} p+i \operatorname{deg}_{y} q$. Therefore, for any choice $r \in \mathbb{N}$ and any choice $c_{0}, \ldots, c_{r} \in C(x)$, we have that $c_{0} f+c_{1} D_{x} f+\cdots+c_{r} D_{x}^{r} f$ is a rational function with denominator $q^{r+1}$ and a numerator whose degree is bounded by $\operatorname{deg}_{y} p+r \operatorname{deg}_{y} q$. Now consider a rational function $g=u / q^{r}$ with $u=u_{0}+u_{1} y+\cdots+u_{s} y^{s} \in C(x)[y]$. Then $D_{y} g=v / q^{r+1}$ for some $v \in C(x)[y]$ of degree at most $s+\operatorname{deg}_{y} q$. In order to get the desired equality $c_{0} f+c_{1} D_{x} f+\cdots+c_{r} D_{x}^{r} f=D_{y} g$, we multiply both sides by $q^{r+1}$ and equate coefficients with respect to $y$. This gives a linear system over $C(x)$ for the variables $c_{0}, \ldots, c_{r}, u_{0}, \ldots, u_{s}$. These are $(r+1)+(s+1)$ variables. The number of equations is at most $1+\max \left(\operatorname{deg}_{y} p+r \operatorname{deg}_{y} q, s+\operatorname{deg}_{q}\right)$, which simplifies to $1+\operatorname{deg}_{y} p+r \operatorname{deg}_{y} q$ if we choose $s=\operatorname{deg}_{y} p+(r-1) \operatorname{deg}_{y} q$. The number of variables exceeds the number of equations if $(r+1)+\left(\operatorname{deg}_{y} p+(r-1) \operatorname{deg}_{y} q+1\right)>1+\operatorname{deg}_{y} p+$ $r \operatorname{deg}_{y} q$, i.e., if $r>\operatorname{deg}(q)-1$. It follows that for $r \geq \operatorname{deg}(q)$ the linear system will have a nontrivial solution. For this nontrivial solution, at least one of $c_{0}, \ldots, c_{r}, u_{0}, \ldots, u_{s}$ is nonzero. It is then not possible that $c_{0}, \ldots, c_{r}$ are all zero, because by our simplifying assumption $g$ is a rational function whose numerator as lower degree than its denominator, so $D_{y} g$ can only be zero if $g$ is zero, and then also $u_{0}, \ldots, u_{s}$ would all have to be zero. We have thus shown that the minimal order telescoper for $f$ has order at $\operatorname{most} \operatorname{deg}(q)$.

The reasoning can be refined such as to also provide bounds for the degrees of the telescopers. This has been done for hyperexponential terms in [27] and for hypergeometric terms in [26]. The resulting curves are simple hyperbolas. However, the degree bounds are not sharp. For the hypergeometric case, also the bit size of the integer coefficients has been analyzed [62]. For general D-finite functions, we know bounds for the order of the telescopers but an order-degree curve has not yet been worked out. Therefore:

Problem 3. Derive an order-degree-curve for general D-finite functions.

It would also be interesting to have bounds for the bit size not only for hypergeometric input but also for other classes, for example for hyperexponential terms.

Problem 4. Derive bounds for the bit size of telescopers for hyperexponential terms.

Experiments show that the order-degree curves following from the analysis of Apagodu-Zeilbergerlike algorithms are not sharp. Better bounds could be obtained if we had a better understanding of the singularities of telescopers. It was shown in [58] how the distinction between removable and non-removable singularities of an operator $L \in C[x]\left[D_{x}\right]$ implies a curve that very accurately describes the degrees of the elements of $\left(C(x)\left[D_{x}\right] L\right) \cap C[x]\left[D_{x}\right]$. Here, a singularity of $L$ is defined as a root of the leading coefficient polynomial (the coefficient of the highest derivative), and such a singularity is called removable if there exists an operator $Q \in C(x)\left[D_{x}\right]$ such that $Q L$ is in $C[x]\left[D_{x}\right]$ and does not have this singularity. The terminology is analogous for recurrence operators, and the connection to order degree curves observed in [58] also applies to this case.

Several algorithms are known for identifying the removable singularities of an operator [8, 3, 30]. Therefore, when a telescoper is known, we obtain a very accurate order-degree curve. However, for the design of efficient creative telescoping algorithms it would be useful to have order-degree curves that can be easily read off from the summand/integrand, rather than from the telescoper. The question therefore is whether it is possible to predict the removable and non-removable singularities of a telescoper directly from the summand/integrand. This leads to the next problem.

Problem 5. (a) Find a way to determine the removable and non-removable singularities of a telescoper for a given proper hypergeometric term $f(n, k)=p c^{n} d^{k} \prod_{i=1}^{m} \Gamma\left(\alpha_{i} n+\beta_{i} k+\gamma_{i}\right)^{e_{i}}(p \in$ $\left.C[n, k], c, d \in C, \alpha_{i}, \beta_{i}, \mathrm{e}_{i} \in \mathbb{N}, \gamma_{i} \in C\right)$, using less computation time than needed for computing a telescoper. 
(b) The analogous question for hyperexponential terms $f(x, y)=\exp (a / b) \prod_{i=1}^{m} p_{i}^{c_{i}}\left(a, b, p_{i} \in\right.$ $\left.C[x, y], c_{i} \in C\right)$.

\section{Differential and Difference Fields}

In the area of differential algebra, a pair $(K, d)$ is called a differential field if $K$ is a field and $d: K \rightarrow K$ is such that $d(a+b)=d(a)+d(b)$ and $d(a b)=d(a) b+a d(b)$ for all $a, b \in K$. For example, the field $K=C(x)$ of rational functions forms a differential field together with the usual derivation $\frac{d}{d x}$. More generally, appropriate differential fields can be used to emulate the behaviour of expressions involving elementary functions under differentiation. The corresponding differential fields are called liouvillean fields. They are used in Risch's integration algorithm [79, 80, 21, 20]. Analogously, a difference field is a pair $(K, s)$ where $K$ is a field and $s: K \rightarrow K$ is such that $s(a+b)=s(a)+s(b)$ and $s(a b)=s(a) s(b)$ for all $a, b \in K$, i.e., $s$ is an automorphism. Difference fields corresponding to liouvillean fields are called $\Pi \Sigma$-fields. They emulate the behaviour of expressions involving nested sums and products under shift and are used in Karr's summation algorithm [59, 60].

The creative telescoping problem can be formulated for differential and difference fields. In the differential case, let $K$ be a field with two derivations $d_{x}, d_{y}: K \rightarrow K$ that commute with each other, and consider the operator algebra $A=K\left[\partial_{x}, \partial_{y}\right]$ with the commutation rules $\partial_{x} \partial_{y}=\partial_{y} \partial_{x}$ and $\partial_{x} a=a \partial_{x}+d_{x}(a)$ and $\partial_{y} a=a \partial_{y}+d_{y}(a)$ for all $a \in K$. Such an operator algebra may act on some function space $F$. For a given $f \in F$ we may then ask, like before, whether there exists $P, Q \in A$ such that $\left(P-\partial_{y} Q\right) \cdot f=0$. Here, $P$ must belong to $K_{x}\left[\partial_{x}\right]$, where $K_{x}=\{u \in K$ : $\left.d_{y}(u)=0\right\}$ is the subfield of $K$ consisting of all elements of $K$ that are constant with respect to $y$. The version for difference fields is analogous.

Schneider [84] was the first to observe that Karr's summation algorithm can be used to solve the creative telescoping problem in very much the same way as Gosper's algorithm is exploited in Zeilberger's algorithm. He has been working on refinements, extensions, and generalizations of summation technology based on difference field theory for many years and has obtained spectacular results, see [87] and the references given there. Yet, some questions have not yet been addressed. In particular, there is no general theory which clarifies under which circumstances a telescoper exists (a question that is settled for the classical hypergeometric case by the work of Abramov et al. $[1,4,2,5])$, or to give a priori bounds on their order or on the cost for their computation. Similar remarks apply in the differential case, for which Raab [76] has recently formulated a creative telescoping approach based on Risch's algorithm, but no theoretical results concerning existence or size of telescopers were given.

Problem 6. For the creative telescoping problem over liouvillean fields (in the differential case) or for $\Pi \Sigma$-fields (in the shift case), derive a criterion for the existence of a telescoper. For the cases where telescopers exist, derive bounds on their order.

In contrast to D-finite functions in the differential case, elementary functions may not have a telescoper. One obstruction to the existence of a telescoper may be the fact that an elementary function can only be elementary integrable if all its residues are constant (cf. Section 5.6 of [21]). A telescoper must therefore at least map all the residues of the given function to constants. This is only possible if the residues are D-finite, which may not be the case. For example, the function $f(x, y)=\frac{x}{\left(\mathrm{e}^{x}-1\right)(1-y)}$ cannot have a telescoper with respect to $y$, because its residue at $y=1$ is $\frac{x}{\mathrm{e}^{x}-1}$, which is not D-finite.

For the shift case, Schneider has an algorithm [85] which computes for a given nested sum expression an equivalent expression in which the nesting depth is as small as possible. This is remarkable because the equivalent representation with minimal depth does usually not belong to the same field in which the input sum is given. So far there is no analogous algorithm for the differential case, although it would be interesting to have one. Therefore:

Problem 7. Design an algorithm which finds for a given expression of nested indefinite integrals an equivalent expression for which the the nesting depth is as small as possible. 
Our last problem in this section relates to the structure of the class of elementary functions. As this class is not closed under integration, the set of elementary integrable elementary functions forms a proper subclass. This class in turn contains integrable as well as non-integrable functions. It is clear that for every $n \in \mathbb{N}$, there is an elementary function which is $n$ times elementary integrable but not $n+1$ times. An example is the $n$th derivative of $\mathrm{e}^{x^{2}}$. On the other hand, there are also elementary functions which can be integrated arbitrarily often without ever leaving the class of elementary functions, for example polynomials. What other functions have this property?

Problem 8. Determine the class of elementary functions with the property that for every $n \in \mathbb{N}$, their $n$-fold integral is again elementary.

Using repeated partial integration, we can show that a function $f(x)$ belongs to this class if and only if for every $n \in \mathbb{N}$ the function $x^{n} f(x)$ is elementary integrable. This implies that all rational functions are arbitrarily often elementary integrable. Note that this is not obvious because the integral of a rational function may involve logarithms of algebraic functions, and such functions need not be elementary integrable.

\section{The Multivariate Case}

While most single sums appearing in practical applications are nowadays no challenge for a computer algebra system, multiple sums may still be too hard. One natural reason is that multiple sums tend to involve expressions in many variables, and such expressions can quickly become too large to be handled efficiently. Another reason is that the algorithms we know for single sums are better than those we know for multiple sums. For single sums, Zeilberger's algorithm supersedes elimination methods such as the so-called Sister Celine algorithm [46, 94, 73]. But while the algorithm of Sister Celine has been generalized to multisums [96, 95], there is no multivariate Zeilberger algorithm yet. We do not even know a multivariate Gosper algorithm.

Problem 9. Develop an algorithm which takes as input a multivariate hypergeometric term $h$ in $m$ discrete variables $k_{1}, \ldots, k_{m}$, and decides whether there exist hypergeometric terms $g_{1}, \ldots, g_{m}$ such that

$$
h=\Delta_{1}\left(g_{1}\right)+\cdots+\Delta_{m}\left(g_{m}\right) .
$$

Here, $\Delta_{i}$ is the forward difference operator with respect to the variable $k_{i}$, i.e., $\Delta_{i} f\left(k_{1}, \ldots, k_{m}\right)=$ $f\left(k_{1}, \ldots, k_{i}+1, \ldots, k_{m}\right)-f\left(k_{1}, \ldots, k_{i}, \ldots, k_{m}\right)$.

A solution of this problem would be an important step towards the development of a Zeilbergerlike algorithm for multisums. Recently, Chen and Singer [31, 32] have given a necessary and sufficient condition for the case when $h$ is a rational function in two variables. Their criterion was then turned into an algorithm by Hou and Wang [54]. In [24] these results were used to derive some conditions on the existence of telescopers for trivariate rational functions. Summability criteria for larger classes, such as the class of hypergeometric terms, may analogously allow for the formulation of existence criteria for telescopers in the multivariate setting. In the long run, we would hope that a multivariate Gosper algorithm serves as a starting point for the development of a reduction-based creative telescoping algorithm for the multivariate setting.

The corresponding question for bivariate rational functions in the differential case has been studied already by Picard [75, 74] many years ago. More recently, Griffiths and Dwork [44, 45, 50, 51] gave a method that works for any number of variables but requires some kind of regularity of the denominator. An algorithm for creative telescoping based on these results was given by Bostan et al. [18].

\section{Binomial Sums}

The principal application of creative telescoping is the construction of recurrence relations satisfied by definite sums. As already indicated in the introduction, such a recurrence can be obtained from 
a telescoper-certificate pair for the summand. However, some care is necessary for this step. In order to be able to sum a relation

$$
c_{0}(n) f(n, k)+\cdots+c_{r}(n) f(n+r, k)=g(n, k+1)-g(n, k)
$$

for $k$ from 0 to $n$, we must assure that the right hand side involving the certificate $g(n, k)$ does not have any poles for the values $k$ in this range. Unfortunately, such poles do appear in examples, and although they usually cancel each other nicely, it is not easy to verify this algorithmically. See [42] for a detailed case study in this context.

For indefinite hypergeometric single sums, Abramov and Petkovsek [7] discuss an alternative to Gosper's algorithm that handles special points properly. Ryabenko [83] gives an accurate summation algorithm for definite sums over a particular class of hypergeometric terms. A continuation of her work towards the full class of hypergeometric terms (or even beyond) would be worthwhile.

Problem 10. Develop an algorithm that correctly transforms a telescoper-certificate pair for a hypergeometric term into a recurrence for the corresponding definite sum. In particular, the algorithm should property take care of any possible issues arising from poles in the certificate.

It appears that the situation is somewhat easier for summands with compact support. A hypergeometric term $f(n, k)$ is said to have compact support if for every $n \in \mathbb{Z}$ there are only finitely many $k \in \mathbb{Z}$ such that $f(n, k)$ is different from zero. In this case, the infinite sum $\sum_{k=-\infty}^{\infty} f(n, k)$ is in fact a terminating sum. For example, we have $\sum_{k=0}^{n}\left(\begin{array}{l}n \\ k\end{array}\right)=\sum_{k=-\infty}^{\infty}\left(\begin{array}{l}n \\ k\end{array}\right)$ because $\left(\begin{array}{l}n \\ k\end{array}\right)=0$ when $k<0$ or $k>n$.

When the sum over $k$ runs through all integers (and there are no issues with poles in the certificate), the transformation of a telescoper-certificate pair to a recurrence for the definite sum is particularly nice. One reason is that the operator $\sum_{k=-\infty}^{\infty}$ commutes with the shift operator $S_{n}$, and therefore, with the telescoper. A second reason is that the right hand side $\sum_{k=-\infty}^{\infty}(g(n, k+1)-g(n, k))$ invariably collapses to zero (because when $f(n, k)$ has compact support, then so does $g(n, k)$ ). Therefore, in the case of compact support, the telescoper for $f(n, k)$ is precisely the recurrence for $\sum_{k} f(n, k)$.

Viewing hypergeometric terms as algebraic objects, it is somewhat unsatisfactory that the concept of compact support is defined "analytically" in terms of the values of sequences associated to the terms. In view of a possible automation, a more algebraic explanation of the phenomenon would be useful. A finite summation operator such as $\Sigma:=\sum_{k=0}^{n}$ does not commute with the shift $S_{n}$. However, if we introduce the evaluation operator $E_{n}$ that acts on bivariate terms by setting $k$ to $n$, then we have the commutation rule $\Sigma S_{n}=S_{n} \Sigma-E_{n} S_{n}$. This rule expresses the fact $\sum_{k=0}^{n} f(n+1, k)=\sum_{k=0}^{n+1} f(n+1, k)-f(n+1, n+1)$. Now consider a telescoper $P=$ $c_{0}(n)+c_{1}(n) S_{n}+\cdots+c_{r}(n) S_{n}^{r}$ with a corresponding certificate $Q$, so that $\left(P-\Delta_{k} Q\right) \cdot f(n, k)=0$. Applying $\Sigma$ to this relation and using the commutation rules leads to

$$
P \Sigma \cdot f(n, k)=\left(\left(c_{1}(n) E_{n} S_{n}+\cdots+c_{r}(n) \sum_{i=1}^{r} S_{n}^{r-i} E_{n} S_{n}^{i}\right)+\left(E_{n} S_{n} Q-E_{0} Q\right)\right) \cdot f(n, k),
$$

where $E_{0}$ denotes an evaluation operator that sets $k$ to 0 . We see that the telescoper $P$ translates directly into an annihilating operator for the sum if and only if the right hand side is zero, i.e., if the operator on the right annihilates the summand. Note that it is irrelevant whether $f(n, k)$ has compact support.

For the differential case, Regensburger, Rosenkranz and collaborators have developed a theory of operator algebras that include both derivations as well as integration operators. Their principal motivation is to solve boundary value problems, see $[81,82,78,52,77]$ and the references given there for an overview of their results. Their algebras also contain evaluation operators similar to the $E_{n}$ introduced above. We would like to see an analogous theory for operator algebras involving summation as well as shift operators.

Problem 11. Develop a theory of operator algebras including shift as well as summation operators, analogous to the theory of Regensburger and Rosenkranz. In this theory, find an algebraic 
explanation why the right hand side of a creative telescoping relation often vanishes for binomial sums.

In a recent paper, Bostan et al. [19] approach the problems related to boundary conditions and possible poles in the certificate from a different direction. Instead of applying creative telescoping directly to the sum in question, they translate the summation problem into an integration problem and apply creative telescoping to this problem. One advantage of this approach is that for the resulting contour integrals there are no problems related to singularities, because the path of integration can always be deformed such as to avoid all the singularities. For this reason, it is not necessary to inspect the certificate, and it is possible to employ efficient algorithms which only compute the telescoper. So far the approach does not apply to all hypergeometric sums but only to a subclass. They call it the class of binomial sums and they show for the case of one variable that a sequence is a binomial sum (in the sense of their definition) if and only if it is the diagonal of a multivariate rational function. The diagonal of a multivariate power series $\sum_{n_{1}, \ldots, n_{r}=0}^{\infty} a_{n_{1}, \ldots, n_{r}} x_{1}^{n_{1}} \cdots x_{r}^{n_{r}}$ is defined as the univariate series $\sum_{n=0}^{\infty} a_{n, n, \ldots, n} x^{n}$. The definition of binomial sums also covers sums with several variables, but no characterization of binomial sums in several variables is given in [19].

Problem 12. Prove or disprove: A multivariate sequence $\left(b_{k_{1}, \ldots, k_{s}}\right)$ in $s$ discrete variables is a binomial sum in the sense of [19] if and only if there exists a rational power series

$$
\sum_{n_{1}, \ldots, n_{r}=0}^{\infty} a_{n_{1}, \ldots, n_{r}} x_{1}^{n_{1}} \cdots x_{r}^{n_{r}}
$$

and $i_{1}, \ldots, i_{s} \in \mathbb{N}$ with $i_{1}+\cdots+i_{s}=r$ such that for all $k_{1}, \ldots, k_{s}$ we have

$$
b_{k_{1}, \ldots, k_{s}}=a_{\underbrace{k_{1}, \ldots, k_{1}}_{i_{1}}}, \underbrace{k_{2}, \ldots, k_{2}}_{i_{2}}, \ldots, \underbrace{k_{s}, \ldots, k_{s}}_{i_{s}}
$$

An important open problem in the context of diagonals is Christol's conjecture [36], which says that every formal power series with integer coefficients and a positive radius of convergence which is the solution of a linear differential equation with polynomial coefficients is the diagonal of some rational power series. In this conjecture, no statement is made about the number of variables of the rational power series. Bostan et al. [19] remark that we must at least allow for three variables, and that no explicit example is known which requires more.

Because of its connection to diagonals, the class of binomial sums as introduced in [19] is not as artificial as it seems at first glance. Nevertheless, also a natural restriction is a restriction. It would be interesting to extend the applicability of the algorithm to a wider class.

Problem 13. Generalize the algorithm of [19] from binomial sums to arbitrary hypergeometric sums.

\section{Nonlinear Equations and Annihilators of Positive Dimension}

In the theory of "holonomic systems" [99], summands and integrands are represented by ideals of operators by which they are annihilated. Properties of the ideal are used to ensure the existence of telescopers and the termination of algorithms. A condition that is typically imposed is that the ideal has Hilbert dimension 0. In this case, the annihilated function is called D-finite. Many functions of practical relevance happen to be D-finite, but it is natural to ask to whether D-finiteness is really needed for creative telescoping to succeed. It turns out that it is not. Already in the 1990s, Majewicz has given a variant of creative telescoping applicable to Abel-type identities [69]. The key observation is that such identities exist because the sum has more than one free variable, and this can compensate for the lack of relations preventing the summand from being D-finite. A summation algorithm by Kauers [61] for sums involving Stirling numbers and an algorithm by Chen and Sun [33] for sums involving Bernoulli numbers are based on similar observations. In 2009, the phenomenon was formulated in more general terms by Chyzak et al. [40]. They showed that telescopers can exist also when the annihilator of the summand/integrand has positive dimension. 
More precisely, consider a function with $n$ free variables and $k$ summation/integration variables, let $I$ be the annihilator of the function and let $T$ be the ideal of telescopers (in the smaller operator algebra corresponding only to the $n$ free variables). Then they show that $\operatorname{dim} T \leq \operatorname{dim} I+(p-1) n$, where $p \in \mathbb{N}$ is a quantity they call the "polynomial growth" of the ideal $I$. Not much is known about this quantity. It seems that we have $p=1$ in most cases of practical interest, but we do not know whether it is connected to more classic quantities defined for (operator) ideals, or even how to compute it for a given ideal $I$.

Problem 14. Clarify the meaning of "polynomial growth" introduced in [40]. Can the definition in [40] be replaced by another one, possibly not equivalent, which also satisfies the bound on $\operatorname{dim} I$ stated above? Is there an efficient algorithm for computing the polynomial growth of a given operator ideal?

For sums involving Stirling numbers, it would also be conceivable to have a creative telescoping algorithm that exploits the special form of their generating function. For example, for the Stirling numbers of the second kind, $\sum_{n, k=0}^{\infty} S(n, k) \frac{x^{n}}{n !} y^{k}=\exp \left(y\left(\mathrm{e}^{x}-1\right)\right)$ is not D-finite but still elementary, so generalized techniques as discussed in Section 4 might apply. The function $f(x, y)=\exp \left(y\left(\mathrm{e}^{x}-1\right)\right)$ is also an example of a function satisfying a system of algebraic differential equations (ADE): we have $f_{x}(x, y)-y f_{y}(x, y)-y f(x, y)=0$ and $f(x, y) f_{y, y}(x, y)-f_{y}(x, y)^{2}=0$. Other prominent examples of non-D-finite functions satisfying algebraic differential equations are the generating function for the partition numbers $\prod_{n=0}^{\infty}\left(1-x^{n}\right)^{-1}$ and the Weierstraß $\wp$-function. Solutions of ADEs also appear in combinatorics, for example as generating functions of certain restricted lattice walks [13].

While there is a reasonably well developed elimination theory for systems of algebraic differential equations [70, 48, 56, 34, 47], no creative telescoping algorithm for this class of functions is known.

Problem 15. Develop a creative telescoping algorithm applicable to functions satisfying systems of ADEs.

For approaching this problem, it may become appropriate to adapt the specification of the creative telescoping problem. In a context where quantities are defined by non-linear equations, it may be too restrictive to require that the telescoper be a linear operator. On the other hand, allowing non-linear operators as telescoper does not seem sensible either as long as the main motivation for creative telescoping is to derive equations for definite integrals, because the application of an integral operator does in general not commute with such an operator. It is a part of the problem to determine a suitable adaption of the creative telescoping problem.

\section{The InVerse Problem}

Using creative telescoping, we can obtain a recurrence satisfied by a given definite sum. The recurrence then serves as a basis for obtaining further information about the sum, such as asymptotic estimates or closed from expressions. The classical application is to use Zeilberger's algorithm in combination with Petkovsek's algorithm [72, 73] in order to decide whether a given definite hypergeometric sum admits a hypergeometric term as a closed form. If the sum comes from some application, there is a certain chance that such a representation exist. However, an arbitrarily chosen sum is not likely to have a closed form. It is even less likely for an arbitrary recurrence (which may or may not come from creative telescoping) to have a hypergeometric closed form. People have therefore designed algorithms for finding more general types of closed form solutions of recurrence equations, for example d'Alembertian solutions [6, 73] or liouvillean solutions [92, 53]. Even more generally, we could ask whether a given recurrence admits a solution that can be expressed as a definite sum. In a way, this would be the inverse problem of creative telescoping. Chen and Singer in [31] gave a characterization of possible linear operator that can be minimal telescopers for bivariate rational functions. However, no algorithm is known for solving this problem in the general case, but it would be very valuable for practical applications. 
Problem 16. Design an algorithm which takes as input a nonzero recurrence operator $P \in$ $\mathbb{Q}[n]\left[S_{n}\right]$ and finds, if at all possible, a bivariate hypergeometric term $f(n, k)$ which has $P$ as a telescoper.

The analogous problems for the differential case and the two mixed cases are interesting as well.

In recent years there has been some activity by van Hoeij and collaborators concerning solutions of recurrences or differential equations in terms of hypergeometric series [93, 23, 22, 67, 57]. In a way, these algorithms solve only special cases of the inverse problem for creative telescoping, thus indicating perhaps that the general problem may be very difficult.

\section{Computational Challenges}

Creative telescoping is not only of theoretical interest but it is also a valuable tool in all contexts where summation and integration problems arise that are beyond the scope of any reasonable hand-calculation. For example, the proof of the qTSPP conjecture [66], which was obtained using Koutschan's Mathematica package [65], involves a creative telescoping problem that leads to a certificate of $4 \mathrm{~Gb}$ size. Such computations are clearly not feasible by hand, and they are also challenging for computers. We shall therefore conclude this paper with two explicit computational challenges which to our knowledge are not feasible by any software currently available.

The first problem is quoted from [63] and concerns the computation of diagonals. Again, the diagonal of a multivariate series $\sum_{n_{1}, \ldots, n_{d}=0}^{\infty} a_{n_{1}, n_{2}, \ldots, n_{d}} x_{1}^{n_{1}} \cdots x_{d}^{n_{d}}$ is defined as $\sum_{n=0}^{\infty} a_{n, n, \ldots, n} x^{n}$. The diagonal of a D-finite series is D-finite [68], and creative telescoping can be used, at least in principle, to derive a recurrence for the diagonal terms $a_{n, n, \ldots, n}$ from a given set of defining equations for the original multivariate series.

Problem 17. For $d=4, \ldots, 12$, prove recurrence equations for the diagonals of the rational series $1 /\left(1-\sum_{i=1}^{d} \frac{x_{i}}{1-x_{i}}\right)$ conjectured in $[63]$.

For $d=1,2$, the problem is easy. For $d=3$, it was solved in [16].

In 2002, Beck and Prixton made an effort to compute the Ehrhart polynomial of Birkhoff polytopes [11], a quantity that is relevant in discrete geometry [12]. There is a Birkhoff polynomial associated to every $n \in \mathbb{N}$. They succeeded in computing the full Ehrhart polynomial for all $n \geq 9$, and the most significant coefficient for the case $n=10$. As a computational challenge, we pose the computation of the full Ehrhart polynomial for $n=10,11,12$. We take advantage of Theorem 2 of [11], where these polynomials are expressed as integrals that are amenable to creative telescoping.

Problem 18. For $n=10,11,12$, compute the polynomial

$$
H_{n}(t)=\frac{1}{(2 \pi i)^{n}} \int_{\left|z_{1}\right|=\epsilon_{1}} \cdots \int_{\left|z_{n}\right|=\epsilon_{n}}\left(z_{1} \cdots z_{n}\right)^{-t-1}\left(\sum_{k=1}^{n} \frac{z_{k}^{t+n-1}}{\prod_{j \neq k}\left(z_{k}-z_{j}\right)}\right)^{n} d z_{n} \cdots d z_{1},
$$

where $0<\epsilon_{1}, \ldots, \epsilon_{n}<1$ are arbitrary.

This problem is similar to the previous one in so far as diagonals can be rephrased as contour integrals. But it is different in that we ask for the polynomials $H_{n}(t)$ rather than for some differential equation satisfied by $H_{n}(t)$. Following the standard approach, we would first use creative telescoping to compute such a differential equation, then determine the space of polynomial solutions of this equation, and then find the unique element of this space that matches the initial terms of $H_{n}(t)$. This element must be $H_{n}(t)$ itself. In the present context, this approach may not be feasible because the computation of the first coefficients of $H_{n}(t)$ is not much easier than the computation of the whole polynomial. So part of the question is whether creative telescoping can help to compute the polynomials directly, without the detour through a differential equation. 


\section{REFERENCES}

[1] Sergei A. Abramov. Applicability of Zeilberger's algorithm to hypergeometric terms. In Proceedings of IS$S A C^{\prime} 02$, pages $1-7,2002$.

[2] Sergei A. Abramov. When does Zeilberger's algorithm succeed? Advances in Applied Mathematics, 30(3):424$441,2003$.

[3] Sergei A. Abramov, Moulay A. Barkatou, and Mark van Hoeij. Apparent singularities of linear difference equations with polynomial coefficients. Applicable Algebra in Engeneering, Communication and Computing, 17(2):117-133, 2006.

[4] Sergei A. Abramov and Ha Q. Le. A criterion for the applicability of Zeilberger's algorithm to a rational function. Discrete Mathematics, 259(1-3):1-17, 2002.

[5] Sergei A. Abramov and Ha Q. Le. On the order of the recurrence produced by the method of creative telescoping. Discrete Mathematics, 298(1-3):2-17, 2005.

[6] Sergei A. Abramov and Marko Petkovsek. D'alembertian solutions of linear differential and difference equations. In Proceedings of ISSAC'94, pages 169-174, 2004.

[7] Sergei A. Abramov and Marko Petkovsek. Gosper's algorithm, accurate summation, and the discrete NewtonLeibniz formula. In Proceedings of ISSAC'05, pages 5-12, 2005.

[8] Sergei A. Abramov and Mark van Hoeij. Desingularization of linear difference operators with polynomial coefficients. In Proceedings of ISSAC'99, pages 269-275, 1999.

[9] Gert Almkvist and Doron Zeilberger. The method of differentiating under the integral sign. Journal of Symbolic Computation, 11(6):571-591, 1990.

[10] Moa Apagodu and Doron Zeilberger. Multi-variable Zeilberger and Almkvist-Zeilberger algorithms and the sharpening of Wilf-Zeilberger theory. Advances in Applied Mathematics, 37(2):139-152, 2006.

[11] Matthias Beck and Dennis Pixton. The Ehrhart polynomial of the Birkhoff polytope. Discrete Computational Geometry, 30:623-637, 2003.

[12] Matthias Beck and Sinai Robins. Computing the Continuous Discretely: Integer-Point Enumeration in Polyhedra. Springer, 2007.

[13] Olivier Bernardi, Mireille Bousquet-Melou, and Kilian Raschel. Counting quadrant walks via Tutte's invariant method. In Proceedings of FPSAC'16, 2016. to appear.

[14] Alin Bostan, Shaoshi Chen, Frédéric Chyzak, and Ziming Li. Complexity of creative telescoping for bivariate rational functions. In Proceedings of ISSAC'10, pages 203-210, 2010.

[15] Alin Bostan, Shaoshi Chen, Frederic Chyzak, Ziming Li, and Guoce Xin. Hermite reduction and creative telescoping for hyperexponential functions. In Proceedings of ISSAC'13, pages 77-84, 2013.

[16] Alin Bostan, Frederic Chyzak, Mark van Hoeij, and Lucien Pech. Explicit formula for the generating series of diagonal 3d rook paths. Seminaire Lotharingien Combinatoire, 66(B66a), 2011.

[17] Alin Bostan, Louis Dumont, and Bruno Salvy. Efficient algorithms for mixed creative telescoping. In Proceedings of ISSAC'16, pages 127-134, 2016.

[18] Alin Bostan, Pierre Lairez, and Bruno Salvy. Creative telescoping for rational functions using the GriffithsDwork method. In Proceedings of ISSAC'13, pages 93-100, 2013.

[19] Alin Bostan, Pierre Lairez, and Bruno Salvy. Multiple binomial sums. Technical Report 1510.07487, ArXiv, 2015.

[20] Manuel Bronstein. Symbolic integration tutorial. ISSAC'98, 1998.

[21] Manuel Bronstein. Symbolic Integration I, volume 1 of Algorithms and Computation in Mathematics. Springer, 2nd edition, 2005.

[22] Yongjae Cha. Closed Form Solutions of Linear Difference Equations. PhD thesis, Florida State University, 2010.

[23] Yongjae Cha, Mark van Hoeij, and Giles Levy. Solving recurrence relations using local invariants. In Proceedings ISSAC'10, pages 303-309, 2010.

[24] Shaoshi Chen, Qing-Hu Hou, George Labahn, and Rong-Hua Wang. Existence problem of telescopers: Beyond the bivariate case. In Proceedings of ISSAC'16, pages 167-174, 2016.

[25] Shaoshi Chen, Hui Huang, Manuel Kauers, and Ziming Li. A modified Abramov-Petkovsek reduction and creative telescoping for hypergeometric terms. In Proceedings of ISSAC'15, pages 117-124, 2015.

[26] Shaoshi Chen and Manuel Kauers. Order-degree curves for hypergeometric creative telescoping. In Proceedings of ISSAC'12, pages 122-129, 2012.

[27] Shaoshi Chen and Manuel Kauers. Trading order for degree in creative telescoping. Journal of Symbolic Computation, 47(8):968-995, 2012.

[28] Shaoshi Chen, Manuel Kauers, and Christoph Koutschan. A generalized apagodu-zeilberger algorithm. In Proceedings of ISSAC'14, pages 107-114, 2014.

[29] Shaoshi Chen, Manuel Kauers, and Christoph Koutschan. Reduction-based creative telescoping for algebraic functions. In Proceedings of ISSAC'16, pages 175-182, 2016.

[30] Shaoshi Chen, Manuel Kauers, and Michael F. Singer. Desingularization of Ore operators. Journal of Symbolic Computation, 74(5/6):617-626, 2016.

[31] Shaoshi Chen and Michael F. Singer. Residues and telescopers for bivariate rational functions. Advances in Applied Mathematics, 49(2):111-133, 2012 
[32] Shaoshi Chen and Michael F. Singer. On the summability of bivariate rational functions. Journal of Algebra, 409:320-343, 2014.

[33] William Y. C. Chen and Lisa H. Sun. Extended Zeilberger's algorithm for identities on Bernoulli and Euler polynomials. Journal of Number Theory, 129(9):2111-2132, 2009.

[34] Yufu Chen and Xiao-Shan Gao. Involutive characteristic sets of algebraic partial differential equation systems. Science in China (A), 46(4):469-487, 2003.

[35] Charles Chevalley. Introduction to the Theory of Algebraic Functions of One Variable. AMS, 1951.

[36] Gilles Christol. Globally bounded solutions of differential equations. In Analytic number theory, volume 1434 of Lecture Notes in Mathematics, pages 45-64, 1990.

[37] Frédéric Chyzak. Fonctions holonomes en calcul formel. PhD thesis, INRIA Rocquencourt, 1998.

[38] Frédéric Chyzak. An extension of Zeilberger's fast algorithm to general holonomic functions. Discrete Mathematics, 217:115-134, 2000.

[39] Frederic Chyzak. The ABC of creative telescoping: Algorithms, bounds, complexity. Memoire d'habilitation a diriger les recherches, 2014

[40] Frederic Chyzak, Manuel Kauers, and Bruno Salvy. A non-holonomic systems approach to special function identities. In John May, editor, Proceedings of ISSAC'09, pages 111-118, 2009.

[41] Frédéric Chyzak and Bruno Salvy. Non-commutative elimination in Ore algebras proves multivariate identities. Journal of Symbolic Computation, 26:187-227, 1998.

[42] Frdric Chyzak, Assia Mahboubi, Thomas Sibut-Pinote, and Enrico Tassi. A computer-algebra-based formal proof of the irrationality of $\zeta(3)$. In Gerwin Klein and Ruben Gamboa, editors, Interactive Theorem Proving, number 8558 in Lecture Notes in Computer Science, pages 160-176, Vienna, Austria, 2014. Springer.

[43] Hao Du, Hui Huang, and Ziming Li. Reduction-based creative telescoping for $q$-hypergeometric terms. in preparation.

[44] Bernhard Dwork. On the zeta function of a hypersurface. Publications Mathematiques de l'IHES, 12:5-68, 1962.

[45] Bernhard Dwork. On the zeta function of a hypersurface II. Annals of Mathematics, 80(2):227-299, 1964.

[46] Sister Mary Celine Fasenmyer. A note on pure recurrence relations. The American Mathematical Monthly, $56: 14-17,1949$.

[47] Xiao-Shan Gao, Wei Li, and Chun-Ming Yuan. Intersection theory in differential algebraic geometry: generic intersections and the differential Chow form. Trans. Amer. Math. Soc., 365(9):4575-4632, 2013.

[48] Vladimir P. Gerdt. Gröbner bases and involutive methods of algebraic and differential equations. Math. Comput. Modeling, 25(8/9):75-90, 1997.

[49] William Gosper. Decision procedure for indefinite hypergeometric summation. Proceedings of the National Academy of Sciences of the United States of America, 75:40-42, 1978.

[50] Philip A. Griffiths. On the periods of certain rational integrals I. Annals of Mathematics, 90(3):460-495, 1969.

[51] Philip A. Griffiths. On the periods of certain rational integrals II. Annals of Mathematics, 90(3):496-541, 1969.

[52] Li Guo, Georg Regensburger, and Markus Rosenkranz. On integro-differential algebras. Journal of Pure and Applied Algebra, 218:456-473, 2014.

[53] Peter A. Hendriks and Michael F. Singer. Solving difference equations in finite terms. Journal of Symbolic Computation, 27(3):239-259, 1999.

[54] Qing-Hu Hou and Ronghua Wang. An algorithm for deciding the summability of bivariate rational functions. Advances in Applied Mathematics, 64(3):31-49, 2015.

[55] Hui Huang. New bounds for creative telescoping. In Proceedings of ISSAC'16, pages 279-286, 2016.

[56] Evelyne Hubert. Factorization-free decomposition algorithms in differential algebra. Journal of Symbolic Computation, 29(4-5):641-662, 2000.

[57] Erdal Imamoglu and Mark van Hoeij. Computing hypergeometric solutions of second order linear differential equations using quotients of formal solutions. In Proceedings ISSAC'15, pages 235-242, 2015.

[58] Maximilian Jaroschek, Manuel Kauers, Shaoshi Chen, and Michael F. Singer. Desingularization explains order-degree curves for Ore operators. In Manuel Kauers, editor, Proceedings of ISSAC'13, pages 157-164, 2013

[59] Michael Karr. Summation in finite terms. Journal of the ACM, 28:305-350, 1981.

[60] Michael Karr. Theory of summation in finite terms. Journal of Symbolic Computation, 1(3):303-315, 1985.

[61] Manuel Kauers. Summation algorithms for Stirling number identities. Journal of Symbolic Computation, 42(11):948-970, 2007.

[62] Manuel Kauers and Lily Yen. On the length of integers in telescopers for proper hypergeometric terms. Journal of Symbolic Computation, 66(1-2):21-33, 2015.

[63] Manuel Kauers and Doron Zeilberger. The computational challenge of enumerating high dimensional rook walks. Advances in Applied Mathematics, 47(4):813-819, 2011.

[64] Christoph Koutschan. A fast approach to creative telescoping. Mathematics in Computer Science, 4(2-3):259266, 2010.

[65] Christoph Koutschan. HolonomicFunctions (User's Guide). Technical Report 10-01, RISC Report Series, University of Linz, Austria, January 2010. 
[66] Christoph Koutschan, Manuel Kauers, and Doron Zeilberger. Proof of George Andrews' and David Robbins' q-TSPP-conjecture. Proceedings of the National Academy of Sciences, 108(6):2196-2199, 2011.

[67] Vijay Jung Kunwar and Mark van Hoeij. Second order differential equations with hypergeometric solutions of degree three. In Proceedings of ISSAC'13, pages 235-242, 2013.

[68] L. Lipshitz. The diagonal of a D-finite power series is D-finite. Journal of Algebra, 113:373-378, 1988.

[69] John E. Majewicz. WZ-style certification and sister Celine's technique for Abel-type identities. J. Difference Eqs and Appl., 2:55-65, 1996.

[70] Elisabeth Mansfield. Differential Gröbner bases. PhD thesis, University of Sydney, 1993.

[71] Mohamud Mohammed and Doron Zeilberger. Sharp upper bounds for the orders of the recurrences outputted by the Zeilberger and q-Zeilberger algorithms. Journal of Symbolic Computation, 39(2):201-207, 2005.

[72] Marko Petkovšek. Hypergeometric solutions of linear recurrences with polynomial coefficients. Journal of Symbolic Computation, 14(2-3):243-264, 1992.

[73] Marko Petkovšek, Herbert Wilf, and Doron Zeilberger. $A=B$. AK Peters, Ltd., 1997.

[74] Emile Picard. Sur les periodes des integrales doubles et sur une classe d'equations differentielles lineaires. Annales scientifiques de l'E.N.S., 50:393-395, 1933.

[75] Emile Picard and Georges Simart. Theorie des fonctions algebriques de deux variables independantes. Gauthier-Villars, 1906.

[76] Clemens Raab. Definite Integration in Differential Fields. PhD thesis, Johannes Kepler University, 2012.

[77] Georg Regensburger. Symbolic computation with integro-differential operators. In Proceedings of ISSAC'16, pages $17-18,2016$.

[78] Georg Regensburger, Markus Rosenkranz, and Johannes Middeke. A skew polynomial approach to integrodifferential operators. In Proceedings of ISSAC'09, pages 287-294, 2009.

[79] Robert H. Risch. The problem of integration in finite terms. Transactions of the American Mathematical Society, 139:167-189, 1969.

[80] Robert H. Risch. The solution of the problem of integration in finite terms. Bulletin of the American Mathematical Society, 79:605-608, 1970.

[81] Markus Rosenkranz and Georg Regensburger. Integro-differential polynomials and operators. In Proceedings of ISSAC'08, pages 261-268, 2008.

[82] Markus Rosenkranz and Georg Regensburger. Solving and factoring boundary problems for linear ordinary differential equations in differential algebras. Journal of Symbolic Computation, 43(8):515-544, 2008.

[83] Anna A. Ryabenko. A definite summation of hypergeometric terms of special kind. Programming and Computer Software, 37(4):187-191, 2011.

[84] Carsten Schneider. Symbolic Summation in Difference Fields. PhD thesis, RISC-Linz, Johannes Kepler Universität Linz, 2001.

[85] Carsten Schneider. Finding telescopers with minimal depth for indefinite nested sum and product expressions. In Proceedings of ISSAC'05, pages 285-292, July 2005.

[86] Carsten Schneider. A refined difference field theory for symbolic summation. Journal of Symbolic Computation, 43:611-644, 2008

[87] Carsten Schneider. Simplifying multiple sums in difference fields. In Johannes Blümlein and Carsten Schneider, editors, Computer Algebra in Quantum Field Theory: Integration, Summation and Special Functions, Texts and Monographs in Symbolic Computation, pages 325-360. Springer, 2013.

[88] Nobuki Takayama. An algorithm of constructing the integral of a module. In Proceedings of ISSAC'90, pages 206-211, 1990.

[89] Nobuki Takayama. Gröbner basis, integration and transcendental functions. In Proceedings of ISSAC'90, pages 152-156, 1990.

[90] Barry M. Trager. On the Integration of Algebraic Functions. PhD thesis, MIT, 1984.

[91] Alfred van der Poorten. A proof that Euler missed... - Apéry's proof of the irrationality for $\zeta(3)$. The Mathematical Intelligencer, 1:195-203, 1979.

[92] Marius van der Put and Michael Singer. Galois Theory of Difference Equations, volume 1666 of Lecture Notes in Mathematics. Springer, 1997.

[93] Mark van Hoeij and Quan Yuan. Finding all Bessel type solutions for linear differential equations with rational function coefficients. In Proceedings of ISSAC'10, pages 37-44, 2010.

[94] Pierre Verbaeten. The automatic construction of pure recurrence relations. ACM Sigsam Bulletin, 8, 1974.

[95] Kurt Wegschaider. Computer generated proofs of binomial multi-sum identities. Master's thesis, RISC-Linz, May 1997.

[96] Herb S. Wilf and Doron Zeilberger. An algorithmic proof theory for hypergeometric (ordinary and $q$ ) multisum/integral identities. Inventiones mathematicae, 108:575-633, 1992.

[97] Herbert S. Wilf and Doron Zeilberger. Rational functions certify combinatorial identities. Journal of the American Mathematical Society, 3:147-158, 1990.

[98] Doron Zeilberger. A fast algorithm for proving terminating hypergeometric identities. Discrete Mathematics, 80:207-211, 1990.

[99] Doron Zeilberger. A holonomic systems approach to special function identities. Journal of Computational and Applied Mathematics, 32:321-368, 1990.

[100] Doron Zeilberger. The method of creative telescoping. Journal of Symbolic Computation, 11:195-204, 1991. 
Shaoshi Chen, KLMM, AMSS, Chinese Academy of Sciences, China.

E-mail address: schen@amss.ac.cn

Manuel Kauers, Institute for Algebra, J. Kepler University Linz, Austria.

E-mail address: manuel.kauers@jku.at 